\title{
Electroencephalographic Findings in Idiopathic Rapid Eye Movement Sleep Behavior Disorder with Objective Cognitive Impairment
}

\author{
Jeehyun Hyun, $M D^{1}$, Jung Kyung Hong, $\mathrm{MD}^{2,3}$, In-Young Yoon, MD, $\mathrm{PhD}^{2,3}$ \\ ${ }^{1}$ Department of Neuropsychiatry, Seoul National University Hospital, Seoul, Korea \\ 'Department of Psychiatry, Seoul National University Bundang Hospital, Seongnam, Korea \\ ${ }^{3}$ Seoul National University College of Medicine, Seoul, Korea
}

Received: November 6, 2020

Revised: February 18, 2021

Accepted: April 4, 2021

\section{Correspondence}

Jung Kyung Hong, MD

Department of Psychiatry,

Seoul National University Bundang Hospital,

82 Gumi-ro 173beon-gil, Bundang-gu,

Seongnam 13620, Korea

Tel +82-31-787-7439

Fax +82-31-787-4058

E-mail hongjk15@gmail.com

ORCID

Jeehyun Hyun

https://orcid.org/0000-0003-0974-8499

Jung Kyung Hong

https://orcid.org/0000-0002-1893-7868

In-Young Yoon

https://orcid.org/0000-0002-3995-8238

(c) This is an Open Access article distributed under the terms of the Creative Commons Attribution Non-Commercial License (https://creativecommons.org/licenses/by-nc/4.0) which permits unrestricted non-commercial use, distribution, and reproduction in any medium, provided the original work is properly cited.

\begin{abstract}
Background and Objective Patients with idiopathic rapid eye movement sleep behavior disorder (iRBD) are at the risk of developing neurodegenerative disorders including mild cognitive impairment (MCI). MCI is prevalent among $\mathrm{RBD}$ patients and is the predictive factor for future dementia. We hypothesized that objective cognitive impairment (OCI) might represent an even earlier phase of cognitive decline and demonstrate an association with early electroencephalographic (EEG) changes in iRBD patients.
\end{abstract}

Methods A total of 263 patients with iRBD were divided into three groups according to their cognitive status that was assessed by neuropsychiatric test and structured interview: iRBD-normal cognition (NC), iRBD-OCI, and iRBD-MCI. Each of the participants underwent a waking quantitative EEG recording.

Results iRBD-OCI showed better cognitive performance than iRBD-MCI, especially in the memory domain $(p=0.001)$. Group effect on EEG power was significant in theta $(p=0.043)$ and alpha range $(\mathrm{p}=0.012)$. iRBD-MCI had higher theta $(\mathrm{p}=0.005$ and $\mathrm{p}=0.012)$ and delta power $(\mathrm{p}=$ 0.005 ), and lower alpha power (all $\mathrm{p}<0.01$ ) compared to iRBD-NC. Although there was a trend of increasing slow-wave activity and decreasing alpha activity from iRBD-NC to iRBD-OCI to iRBD$\mathrm{MCI}$, the EEG power of iRBD-OCI did not significantly differ with either side.

Conclusions iRBD patients with OCI may show early changes in EEG activity. Clinicians should recognize the clinical significance of $\mathrm{OCI}$ in the $\mathrm{iRBD}$ population and consider conducting neuropsychological tests even before the presence of the subjective experience of cognitive decline to detect the early stage of cerebral dysfunction and cognitive impairment.

Sleep Med Res 2021;12(1):20-27

Key Words REM sleep behavior disorder, Electroencephalography, Spectral analysis, Mild cognitive impairment, Synucleinopathies.

\section{INTRODUCTION}

Rapid eye movement sleep behavior disorder (RBD) is characterized by an enactment of vivid dreams accompanying the failure of atonia during the rapid eye movement (REM) stage sleep [1]. Generally, the prevalence of RBD has been reported as $0.38-2.01 \%$, but a much higher incidence has been stated in patients with neurodegenerative diseases, especially synucleinopathies [2]. RBD without signs of neurologic disorders is called idiopathic RBD (iRBD). iRBD usually precedes by several years of the clinical onset of the neurodegenerative disease [3]. More than $80 \%$ of patients with iRBD eventually develop a neurodegenerative disease, such as dementia with Lewy body (DLB) or Parkinson's disease (PD) [4]. Mild cognitive impairment (MCI) occurs in approximately half of iRBD patients, and its status in subjects with iRBD strongly predicts 
conversion to dementia related to synucleinopathy $[5,6]$.

Electroencephalogram (EEG) is considered a useful tool for detecting early signs of cerebral dysfunction. There have been several studies investigating EEG in iRBD patients. Generally, increased power of slow waves has been consistently observed in iRBD compared with healthy controls [7-9]. EEG slowing reflects cortical dysfunction and has been observed in various neurodegenerative disorders including Alzheimer's disease $(\mathrm{AD}), \mathrm{PD}$, and DLB [10-13]. Also, the previous studies have found that iRBD patients with MCI had even more increased power of slow waves compared with iRBD patients without MCI $[8,9]$. In another study, a correlation between the EEG powers and the scores of cognitive scale in iRBD patients has been reported, revealing that the patients with more cognitive impairment had more increased power of theta and delta bands [14]. Therefore, the presence of cognitive decline seems to be closely related to the increased power of slow waves in EEG of iRBD patients.

It should be noted that iRBD patients without MCI also showed EEG alterations compared to healthy people $[8,9,15]$. Higher delta, theta, alpha, and beta power have been observed in iRBD patients without MCI compared with the normal controls $[8,9,15]$. However, 'iRBD without MCI' may be an obscure group, with an MCI-centered view. The group 'iRBD without MCI' may involve patients with objective cognitive impairment (OCI) but without any subjective cognitive complaints, given the fact that a diagnosis of MCI requires a subjective cognitive complaint. In the previous studies, only those iRBD patients with subjective complaints were referred to neuropsychological (NP) tests [8] or diagnosed with MCI $[9,15]$ and iRBD patients with OCI have never been studied.

In a general population, though controversial, attention has been given to the clinical significance of OCI. First of all, an inconsistent relationship between subjective memory complaints and objective memory performance in MCI has been reported [16]. Also, the inclusion of subjective memory complaint as diagnostic criteria of MCI has been referred to as its possible contribution to under-diagnosis, by excluding the subjects who truly have cognitive impairment with objective evidence but have few or no subjective concerns due to decreased awareness or overlooking their cognitive decline [16,17]. Finally, there was a study reporting $\mathrm{OCI}$ as an independent predictor of future dementia in elderly women [18]. For the population with iRBD, which itself is an early stage of neurodegeneration, OCI should not be underestimated and grouped under normal cognition (NC). Furthermore, the EEG changes in $\mathrm{RBD}$ patients without MCI might represent an early sign of brain dysfunction due to either OCI or iRBD itself.

In this study, we aimed to further clarify whether there would be a different EEG pattern between iRBD patients with OCI, with $\mathrm{MCI}$, and with NC. We hypothesized that iRBD patients with OCI, which probably presents a lesser degree of cognitive impairment than MCI, might show EEG alterations compared to
iRBD patients with NC, but to a lesser extent compared to EEG changes in iRBD patients with MCI.

\section{METHODS}

\section{Participants}

Individuals with RBD were recruited from the sleep clinic at Seoul National University Bundang Hospital (SNUBH) from April 2014 to May 2019. All the participants underwent polysomnography (PSG) and were diagnosed with RBD by a sleep expert in SNUBH according to the standard criteria of the International Classification of Sleep Disorders-second edition [19]. After the diagnosis, a neurological examination by a neurologist and a NP test were performed. Patients who had degenerative disorders such as $\mathrm{PD}$, multiple system atrophy, $\mathrm{AD}$, and $\mathrm{DLB}$ were excluded from the study. None of the participants showed narcolepsy, a history of major psychiatric disorders, substance use disorders, or any history of brain injury. All the participants were informed of the purpose and procedures of this study and gave written consent. The present study was approved by the Institutional Review Board of SNUBH (B-1408/264-004).

\section{Polysomnography}

All the subjects underwent overnight full PSG using the Embla $^{\text {TM }}$ N7000 device (Embla, Reykjavik, Iceland) with standard electrodes and sensors. In detail, EEG electrodes were applied at F4-A1, F3-A2, C4-A1, C3-A2, O1-A2, and O2-A1 according to the International 10-20 System of Electrode Placement. Electromyogram electrodes were applied at the submentalis, flexor digitorum superficialis, and both the anterior tibialis muscles. Strain gages were used for recording chest and abdominal respiratory movements and nasal pressure cannulas were used to record airflow. Electrocardiogram was applied and oxygen saturation was measured using a pulse oximeter applied to the index finger. Sleep was scored at every 30 s epoch of the nocturnal PSG, and sleep stages were scored visually according to current criteria with allowance for REM sleep without atonia (RSWA) [20]. RSWA was strictly defined using the American Academy of Sleep Medicine manual [20].

\section{Neuropsychological Evaluation and Grouping}

Each participant underwent a structured NP test by trained research neuropsychologists at SNUBH. Cognitive function was assessed based on the Korean version of the Consortium to Establish a Registry for Alzheimer's Disease Assessment Packet Neuropsychological Assessment Battery (CERAD-K-N) [21,22], digit span test [23], Stroop color and word test (SCWT) [24], and frontal assessment battery (FAB) [25]. The CERAD-K-N consists of nine tests: verbal fluency test, 15-item Boston naming test (BNT), Mini-Mental Status Examination for dementia screening (MMSE-DS) [26], word list memory test (WLMT), 
constructional praxis test (CPT), word list recall test (WLRT), word list recognition test (WLRcT), constructional recall test (CRT), and trail making test (TMT). The raw scores of each test were transformed into z-scores which were adjusted for age, sex, and education. Any $z$-score below -1.5 was defined as a significant defect. The geriatric depression scale (GDS) was used to assess depression. A face-to-face standardized diagnostic interview with the geriatric psychiatrists was conducted to obtain a final diagnosis about their cognitive status. Participants turning out to be dementic were excluded from the study.

Individuals diagnosed with $\mathrm{MCI}$ were grouped into $\mathrm{iRBD}$ with MCI (iRBD-MCI) when they met the Consensus Criteria from the International Working Group on MCI [27]: 1) a person is neither normal nor fulfilling diagnostic criteria for dementia, 2) functional activities of the person are mainly preserved, and 3) the person should have evidence of cognitive decline, measured either by self and/or informant report along with the defects in objective cognitive tasks. Those who showed objective evidence of cognitive impairment without subjective complaints were grouped into iRBD with OCI (iRBD-OCI). The rest of the participants who did not show any cognitive deficits from the NP test or the subjective awareness were grouped into iRBD with NC (iRBD-NC).

\section{Resting-State Quantitative EEG}

Waking EEG was recorded with the subjects in a sitting position for 15 minutes during which the subjects were instructed to close their eyes and relax. Participants were monitored by the examiner to check and prevent drowsiness and were asked to open their eyes for 1 minute in the middle. Electrodes were placed according to the extended international $10-20$ system. EEG signals were amplified and digitalized with a 64-channel Neuroscan Synamps (Compumedics, Charlotte, NC, USA) at a sampling rate of $1 \mathrm{kHz}$. EEG data were processed using Neuroguide (NeuroGuide, Applied Neuroscience, Inc., St. Petersburg, FL, USA). The high pass filter was set to $100 \mathrm{~Hz}$ with the low pass filter set to $0.3 \mathrm{~Hz}$. An artifact-free 120-s EEG recording with the eyes closed (24 Epochs of 5-s EEG segments) was selected by visual analysis. Excluded artifacts comprised muscle activity, small body movements, eyelid movements, and micro-sleep. Spectral analysis was done by the fast Fourier transform to compute absolute and relative power of delta $(1.0-4.0 \mathrm{~Hz})$, theta $(4.0-8.0 \mathrm{~Hz})$, alpha $\left(8.0^{-}\right.$ $12.0 \mathrm{~Hz})$, and beta $(12.0-25.0 \mathrm{~Hz})$ bands. The relative power values were the percentages of power of each band in total power. The electrodes were grouped into five cerebral regions and the mean values of power for each region were calculated. As there was no significant asymmetry in any of the five bands, we averaged left and right electrodes. The five cerebral regions investigated were the frontal (FP1, FP2, F3, F4, F7, and F8), central (C3 and $\mathrm{C} 4$ ), parietal (P3 and $\mathrm{P} 4)$, temporal (T3, T4, T5, and T6), and occipital (O1 and $\mathrm{O} 2)$ regions. In case of damaged lead due to excessive artifacts, it was individually removed through visual inspection. All the EEG analyses were blinded to the participants.

\section{Statistical Analyses}

Between-group differences in demographic, clinical, and PSG variables, and NP tests were assessed by analyses of variance. Logistic regression was used to evaluate the risk factors of MCI. Analyses of covariance were used to adjust the covariates. For EEG data, absolute power was natural log-transformed to normalize the data distribution. We used generalized estimating equations (GEEs) to analyze three groups, five region factors, and their interactions in each frequency band [28]. Previously, GEEs have been used in quantitative EEG analyses [29-31].

Statistical significance was set at $\mathrm{p}<0.05$. SPSS version 19.0 for Windows (IBM Corp., Armonk, NY, USA) was used for the analysis. We used Bonferroni-corrected post hoc comparisons for the three groups to determine specific group differences $(\mathrm{p}<0.0167)$.

\section{RESULTS}

\section{Demographic, Clinical, and Polysomnographic Data}

The process of including participants in the present study is summarized in Fig. 1. Among 300 patients diagnosed with RBD at baseline, 263 participants were analyzed. Sixty-eight participants (mean age $69.9 \pm 7.4$ years, $60.3 \%$ male) met the criteria of MCI (iRBD-MCI), 30 patients (mean age $69.0 \pm 7.0$ years, $57.3 \%$ male) had OCI without subjective memory complaints (iRBDOCI), and 165 participants (mean age $66.3 \pm 7.6$ years, $67.3 \%$ male) had normal cognitive function (iRBD-NC).

Demographic, clinical, and polysomnographic variables between the groups are presented in Table 1. There were no significant differences in sex distribution, BMI, and the rate of moderate to severe obstructive sleep apnea [apnea-hypopnea index $(\mathrm{AHI}) \geq 15]$. The iRBD-MCI group had significantly older age, shorter education years, and a higher average score of GDS compared to iRBD-NC. Age $(p=0.011)$ and GDS $(p=0.002)$ were confirmed as the risk factors of MCI from logistic regression analysis conducted in this study sample. Regarding the subtypes of the cognitive impairment, the majority $(64.7 \%)$ of the iRBDMCI group had defects in amnestic domains, while a half of the iRBD-OCI group showed intact memory with impairments on non-amnestic domains only. However, the group difference in the distribution of subtypes of cognitive impairment was not statistically significant.

\section{Neuropsychological Data}

The details of the comparison of the scores of the NP tests are shown in Table 2. The average MMSE score of our entire sample was $27.05 \pm 2.54$ (range: 16 to 30 ).

The iRBD-MCI group showed significantly lower mean z-scores compared to iRBD-NC in all the tests except the CPT. The mean $\mathrm{z}$-scores of $\mathrm{iRBD}$ with the OCI group were generally between 


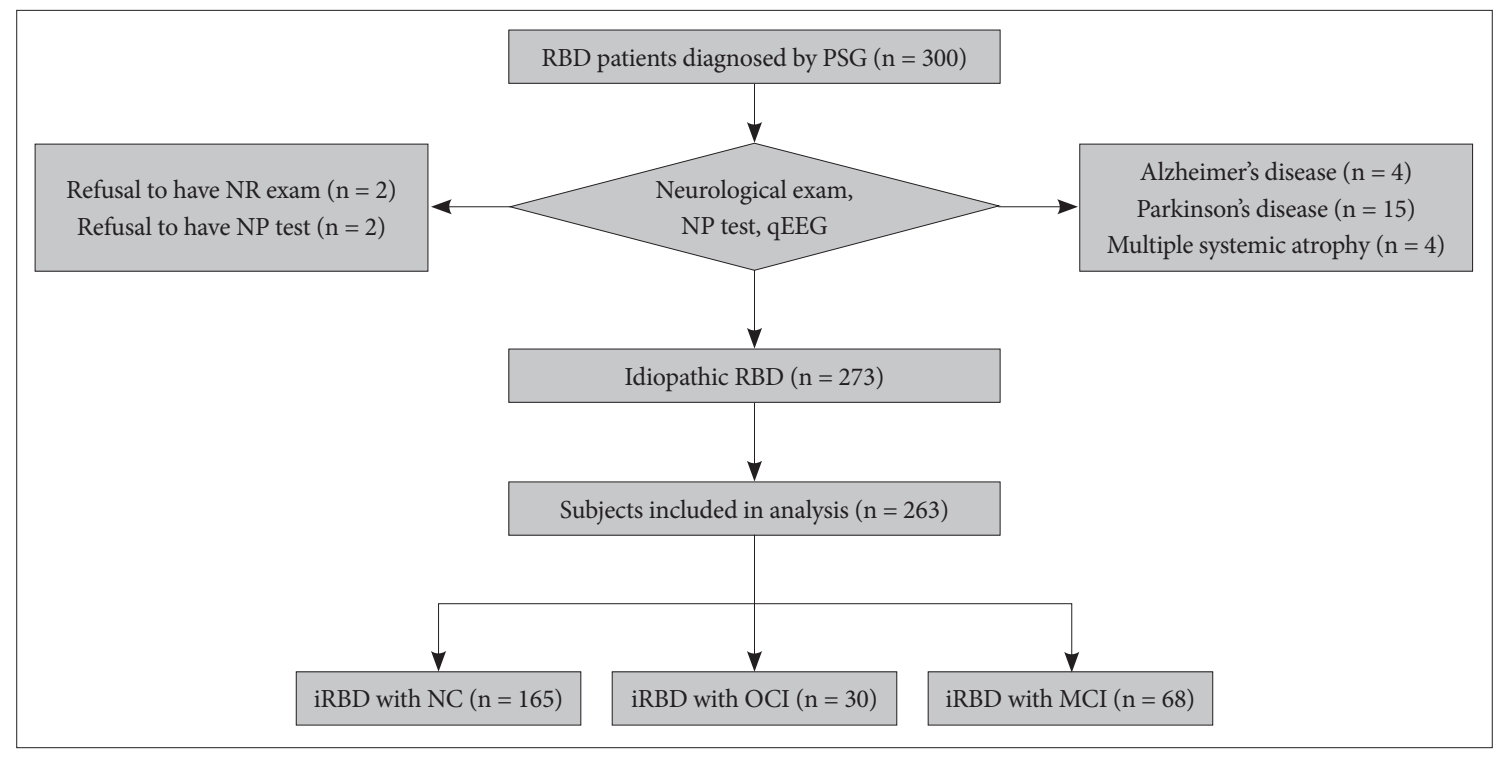

Fig. 1. Flowchart of participant recruitment. RBD: REM sleep behavior disorder, PSG: polysomnography, NP test: neuropsychological test, qEEG: quantitative electroencephalography, NR exam: neurological exam, iRBD: idiopathic RBD, NC: normal cognition, OCl: objective cognitive impairment, $\mathrm{MCl}$ : mild cognitive impairment.

Table 1. Demographic, clinical, and polysomnographic variables in iRBD patients with $\mathrm{NC}, \mathrm{OCl}$, and $\mathrm{MCl}$

\begin{tabular}{|c|c|c|c|c|c|c|}
\hline & $\begin{array}{l}\text { iRBD with NC } \\
\quad(\mathrm{n}=165)\end{array}$ & $\begin{array}{l}\text { iRBD with OCI } \\
\quad(\mathrm{n}=30)\end{array}$ & $\begin{array}{l}\text { iRBD with MCI } \\
\quad(n=68)\end{array}$ & $\mathrm{F}$ & p-value & Post hoc \\
\hline Age (years) & $66.29 \pm 7.56$ & $69 \pm 7.13$ & $69.85 \pm 7.4$ & 6.131 & 0.003 & $\mathrm{NC}<\mathrm{MCI}$ \\
\hline Male & $111(67.3)$ & $17(56.7)$ & $41(60.3)$ & 0.931 & 0.395 & \\
\hline Education years & $12.9 \pm 4.5$ & $11.57 \pm 5.41$ & $10.81 \pm 4.13$ & 5.514 & 0.005 & $\mathrm{NC}>\mathrm{MCI}$ \\
\hline BMI & $24.63 \pm 3.06$ & $25.21 \pm 3.93$ & $24.68 \pm 2.4$ & 0.460 & 0.632 & \\
\hline $\mathrm{AHI} \geq 15$ & $45(27.3)$ & $10(33.3)$ & $19(27.9)$ & 0.229 & 0.795 & \\
\hline GDS score & $7.74 \pm 6.01$ & $7.5 \pm 6.69$ & $10.9 \pm 7.36$ & 6.112 & 0.003 & $\mathrm{NC}<\mathrm{MCI}$ \\
\hline Subtypes of cognitive impairments & & & & & 0.516 & \\
\hline Amnestic, single & & $8(26.7)$ & $25(36.8)$ & & & \\
\hline Amnestic, multiple & & $7(23.3)$ & $19(27.9)$ & & & \\
\hline Nonamnestic, single & & $10(33.3)$ & $18(26.5)$ & & & \\
\hline Nonamnestic, multiple & & $5(16.7)$ & $6(8.8)$ & & & \\
\hline \multicolumn{7}{|l|}{ Polysomnography variables } \\
\hline Total sleep time, min & $354.80 \pm 59.48$ & $355.00 \pm 74.64$ & $340.80 \pm 69.00$ & 1.221 & 0.297 & \\
\hline Stage N1 sleep, \% & $10.72 \pm 8.07$ & $9.11 \pm 4.18$ & $11.38 \pm 7.02$ & 0.967 & 0.381 & \\
\hline Stage N2 sleep, \% & $43.26 \pm 11.38$ & $47.10 \pm 11.56$ & $41.71 \pm 12.24$ & 2.238 & 0.109 & \\
\hline Stage N3 sleep, \% & $9.04 \pm 7.79$ & $8.69 \pm 8.03$ & $9.48 \pm 9.72$ & 0.108 & 0.897 & \\
\hline Stage REM, \% & $17.05 \pm 6.42$ & $14.43 \pm 5.93$ & $16.07 \pm 8.07$ & 2.049 & 0.131 & \\
\hline Sleep onset latency, min & $26.86 \pm 32.13$ & $27.98 \pm 31.20$ & $28.19 \pm 33.95$ & 0.047 & 0.954 & \\
\hline Sleep efficiency, $\%$ & $74.08 \pm 11.96$ & $72.96 \pm 14.53$ & $72.02 \pm 12.95$ & 0.678 & 0.509 & \\
\hline Wake after sleep onset, min & $96.84 \pm 56.13$ & $104.45 \pm 75.58$ & $107.80 \pm 64.79$ & 0.853 & 0.427 & \\
\hline AHI & $11.81 \pm 13.48$ & $13.71 \pm 14.86$ & $10.20 \pm 11.26$ & 0.797 & 0.452 & \\
\hline PLMI & $19.16 \pm 33.71$ & $18.13 \pm 29.62$ & $27.23 \pm 31.50$ & 1.607 & 0.203 & \\
\hline
\end{tabular}

Data are presented as mean \pm standard deviation or $\mathrm{n}(\%)$.

iRBD: idiopathic REM sleep behavior disorder, NC: normal cognition, OCI: objective cognitive impairment, MCI: mild cognitive impairment, BMI: body mass index, AHI: apnea-hypopnea index, GDS: geriatric depression scale, REM: rapid eye movement sleep, PLMI: periodic limb movement index. 
Table 2. Comparison of z-scores of neuropsychological tests between $\mathrm{RBD}$ patients with $\mathrm{NC}, \mathrm{OCl}$, and $\mathrm{MCl}$

\begin{tabular}{|c|c|c|c|c|c|c|c|c|c|}
\hline & \multicolumn{2}{|c|}{$\begin{array}{c}\text { iRBD with NC } \\
\quad(\mathrm{n}=165)\end{array}$} & \multicolumn{2}{|c|}{$\begin{array}{l}\text { iRBD with OCI } \\
\quad(\mathrm{n}=30)\end{array}$} & \multicolumn{2}{|c|}{$\begin{array}{l}\text { iRBD with MCI } \\
\quad(\mathrm{n}=68)\end{array}$} & \multirow[t]{2}{*}{$\mathrm{F}$} & \multirow[t]{2}{*}{$\mathrm{p}$-value } & \multirow[t]{2}{*}{ Post hoc } \\
\hline & Mean & $\mathrm{SD}$ & Mean & SD & Mean & SD & & & \\
\hline $\operatorname{MMSE}(\mathrm{raw})^{*}$ & 27.70 & 2.02 & 27.10 & 1.90 & 25.50 & 3.15 & 20.487 & $<0.001$ & $\mathrm{NC}>\mathrm{MCI}$ \\
\hline MMSE* & 0.20 & 1.00 & 0.06 & 0.86 & -0.63 & 1.43 & 14.012 & $<0.001$ & $\mathrm{NC}>\mathrm{MCI}$ \\
\hline \multicolumn{10}{|l|}{ Attention } \\
\hline Digit Span Forward & 0.54 & 1.01 & 0.24 & 1.15 & -0.18 & 0.80 & 12.203 & $<0.001$ & $\mathrm{NC}>\mathrm{MCI}$ \\
\hline Digit Span Backward & 0.61 & 1.28 & -0.05 & 0.89 & -0.34 & 0.85 & 17.469 & $<0.001$ & $\mathrm{NC}>\mathrm{OCI}, \mathrm{MCI}$ \\
\hline \multicolumn{10}{|l|}{ Memory } \\
\hline Word list memory test & 0.52 & 0.82 & -0.09 & 0.98 & -0.47 & 0.81 & 33.322 & $<0.001$ & $\mathrm{NC}>\mathrm{OCI}, \mathrm{MCI}$ \\
\hline Word list recall test & 0.10 & 0.82 & -0.42 & 1.15 & -1.15 & 0.95 & 43.658 & $<0.001$ & $\mathrm{NC}>\mathrm{OCI}>\mathrm{MCI}$ \\
\hline Word list recognition test & 0.12 & 0.64 & -0.26 & 0.97 & -1.06 & 1.37 & 37.593 & $<0.001$ & $\mathrm{NC}, \mathrm{OCI}>\mathrm{MCI}$ \\
\hline Constructional recall test & 0.31 & 0.89 & -0.33 & 1.04 & -0.55 & 1.07 & 19.812 & $<0.001$ & $\mathrm{NC}>\mathrm{OCI}, \mathrm{MCI}$ \\
\hline \multicolumn{10}{|l|}{ Visuospatial functions } \\
\hline Constructional praxis test & 0.20 & 0.61 & -0.25 & 1.05 & -0.05 & 0.93 & 5.734 & 0.003 & $\mathrm{NC}>\mathrm{OCI}$ \\
\hline \multicolumn{10}{|l|}{ Language } \\
\hline Categorical fluency test & 0.55 & 1.13 & 0.04 & 0.86 & -0.15 & 1.00 & 9.771 & $<0.001$ & $\mathrm{NC}>\mathrm{MCI}$ \\
\hline Boston naming test & 0.81 & 0.70 & 0.39 & 0.96 & 0.27 & 1.02 & 10.390 & $<0.001$ & $\mathrm{NC}>\mathrm{OCI}, \mathrm{MCI}$ \\
\hline \multicolumn{10}{|l|}{ Frontal functions } \\
\hline Frontal assessment battery & 0.38 & 0.63 & -0.23 & 1.05 & -0.16 & 0.85 & 18.053 & $<0.001$ & $\mathrm{NC}>\mathrm{OCI}, \mathrm{MCI}$ \\
\hline Trail-making test A & 1.02 & 0.48 & 0.68 & 0.74 & 0.48 & 0.95 & 16.693 & $<0.001$ & $\mathrm{NC}>\mathrm{OCI}, \mathrm{MCI}$ \\
\hline Stroop test word-color & 0.42 & 1.00 & -0.60 & 1.12 & -0.38 & 0.93 & 22.293 & $<0.001$ & $\mathrm{NC}>\mathrm{OCI}, \mathrm{MCI}$ \\
\hline
\end{tabular}

$*_{\mathrm{n}}=159$ for iRBD with NC.

iRBD: idiopathic REM sleep behavior disorder, MMSE: Mini-Mental Status Examination, NC: normal cognition, OCI: objective cognitive impairment, MCI: mild cognitive impairment.

iRBD-MCI and iRBD-NC. The significant difference between iRBD-OCI and iRBD-NC was revealed in attention (digit span backward, $\mathrm{p}=0.011$ ), verbal memory (WLMT, $\mathrm{p}=0.001$; WLRT, $\mathrm{p}=0.012$ ), visuospatial memory and construction (CRT, $\mathrm{p}=$ 0.003; CPT, $\mathrm{p}=0.009)$, language (BNT, $\mathrm{p}=0.031$ ), and frontal executive function (FAB, $\mathrm{p}<0.001$; TMT-A, $\mathrm{p}=0.032$; SCWT, $\mathrm{p}<0.001$ ). Between iRBD-OCI and iRBD-MCI, a significant difference was observed only in the memory domain (WLRT, $\mathrm{p}=$ 0.001; WLRcT, $\mathrm{p}<0.001$ ). The $\mathrm{z}$-scores of digit span forward and categorical fluency in the iRBD-OCI group were not significantly different from either group. The group difference of the NP performance did not change after the GDS score was adjusted as a covariate.

\section{Comparison of EEG between the Groups}

Age, sex, GDS score, and AHI were adjusted for the EEG analyses while the adjustment of each covariate did not influence the significance of the results. The group-by-region effect was not significant for the absolute EEG power in any band. There was no statistically significant difference for the absolute power of any band in any brain region between the groups.

Regarding the relative EEG power, there was no significant group-by-region interaction $\left(\chi^{2}=10.29, \mathrm{p}=0.245\right)$ or group ef- fect $\left(\chi^{2}=5.71, p=0.058\right)$ for the delta band. However, delta power in the parietal region was higher in the iRBD-MCI group compared to the iRBD-NC group ( $p=0.005$ ). Regarding the theta power, there was no significant group-by-region interaction $\left(\chi^{2}\right.$ $=8.80, \mathrm{p}=0.359)$ but a significant main group effect $\left(\chi^{2}=6.32\right.$, $\mathrm{p}=0.043)$ was noted. In the post hoc test, the iRBD-MCI group showed higher relative theta power in the frontal $(\mathrm{p}=0.005)$ and temporal $(\mathrm{p}=0.012)$ areas. For alpha power, group-by region interaction was not significant $\left(\chi^{2}=7.33, \mathrm{p}=0.502\right)$ but there was the significant main group effect $\left(\chi^{2}=8.90, \mathrm{p}=0.012\right)$. The post hoc test revealed that the iRBD-MCI group had lower relative alpha power in frontal $(\mathrm{p}=0.005)$, temporal $(\mathrm{p}=0.003)$, parietal $(\mathrm{p}=0.002)$, and occipital $(\mathrm{p}=0.002)$ areas than the iRBD-NC group. For the beta power, no group-by-region interaction $\left(\chi^{2}=\right.$ 9.41, $\mathrm{p}=0.309)$ or group effect $\left(\chi^{2}=0.01, \mathrm{p}=0.994\right)$ was found to be significant and no group difference was observed for any band in any region.

As shown in Fig. 2, the spectral EEG power of each band in the iRBD-OCI group was mainly between the EEG power in the iRBD-NC group and in the iRBD-MCI group. However, the EEG power in the iRBD-OCI group did not significantly differ from the iRBD-NC group or the iRBD-MCI group for all the bands and areas. 


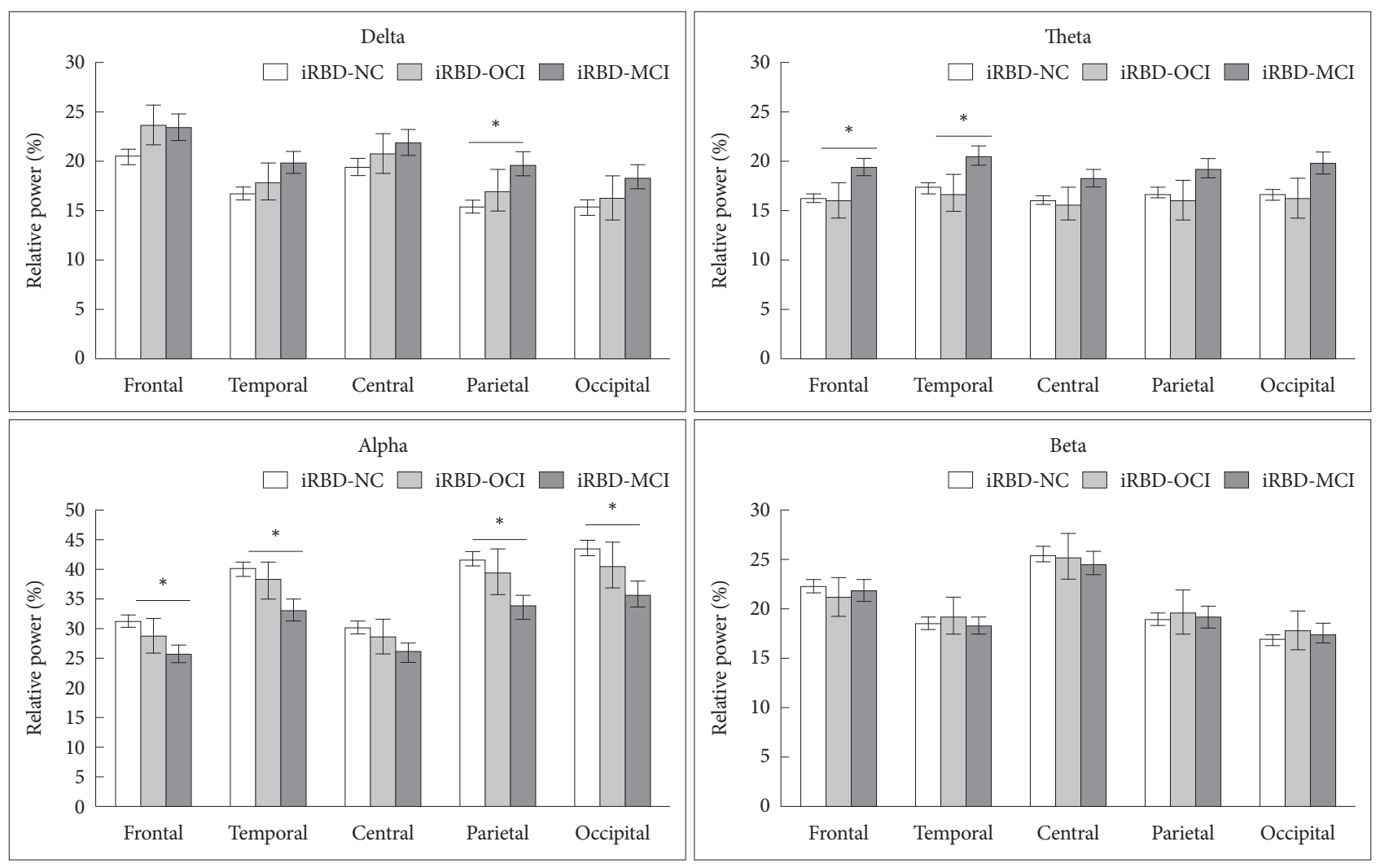

Fig. 2. Comparison of relative electroencephalographic spectral power between the three groups. There was a stepwise change in the order of $\mathrm{NC}-\mathrm{OCl}-\mathrm{MCl}$, although the $\mathrm{OCl}$ group did not demonstrate a significant difference with any other group. *Significant difference between iRBD-NC and iRBD-MCl was revealed in the post hoc test with the Bonferroni correction $(p<0.0167)$. The vertical bar shows the standard errors. iRBD: idiopathic REM sleep behavior disorder, NC: normal cognition, OCl: objective cognitive impairment, MCl: mild cognitive impairment.

\section{DISCUSSION}

Our study attempted to draw clinical attention to OCI in iRBD, which may precede MCI. The spectral power of waking quantitative EEG was compared between the three iRBD groups, each with NC, OCI, and MCI according to standard clinical evaluation and NP tests. Significant differences were observed in EEG activity between the iRBD-NC group and iRBD-MCI group. Comprehensively, iRBD with MCI group showed higher delta power in the parietal region and theta power in frontal and temporal regions, and lower alpha power in frontal, temporal, parietal, and occipital regions compared to iRBD with NC group. The EEG power of each band in the iRBD-OCI group was mostly between the power of the $\mathrm{NC}$ and the MCI groups.

The results of the increased slow waves and decreased alpha activity in the iRBD-MCI group compared to the iRBD-NC group were largely in agreement with the results of a previous study. The only comparable cross-sectional study by Rodrigues Brazète et al [9]. also used waking quantitative EEG, but divided iRBD patients into [+] MCI and [-] MCI. The iRBD [+] MCI group had higher relative theta power in the temporal, parietal, and occipital areas, and lower alpha power in the occipital area than the iRBD [-] MCI group. The between-group difference was present in similar EEG frequency (i.e., theta and alpha), while the affected regions appeared to be slightly different from the observations of the present study. In our study, the difference between MCI and NC groups in the theta power was limited to the frontal and temporal regions and the group difference in the alpha power was observed across all the brain regions except the central area. The group difference in both theta and alpha power covered the frontal area. Considering that the iRBD [-] MCI group in Brazete's study was equivalent to the iRBD-OCI group plus the iRBD-NC group in the current study, iRBD-NC was a more refined subgroup instead of merely negative to MCI. The additional affected area (frontal region) and EEG frequency (alpha band) in this study may be attributed to the exclusion of the iRBD-OCI group compared to the previous study, although the different sample size, quantitative EEG protocol, and analytic method might also play a pivotal role.

The iRBD-OCI group did not show any significant statistical difference compared to either the iRBD-MCI or the iRBD-NC group. A significant difference in EEG activity, particularly within the delta, theta, and alpha ranges, was seen only between the iRBD-MCI and the iRBD-NC groups. We carefully suggested that iRBD patients with OCI may have EEG activity in the middle between iRBD-MCI and iRBD-NC and not incline to either 
side so that the difference with either group was hard to reach statistical significance. As can be seen in Fig. 2, EEG change seemed to be parallel to the degree of cognitive decline, from $\mathrm{NC}$ to OCI to $\mathrm{MCI}$, which is particularly apparent in increasing delta and decreasing alpha activity. Iranzo et al. [8] has already suggested the possibility of a gradual change in EEG activity concurrent with the development of cognitive decline in iRBD patients, despite a completely different study design. Iranzo et al. [8] recruited 23 iRBD patients who did not show any cognitive impairment at baseline and then divided them into two subgroups according to their later development of MCI. After the mean followup duration of 2.4 years, 10 had NC (iRBD) and 13 iRBD patients newly developed MCI (iRBD + MCI) with a difference in EEG activity at baseline. Patients who developed MCI later demonstrated more increased slow-wave activity at baseline. What is in line with our study is the conclusion that EEG change started earlier than obvious cognitive impairment in $\mathrm{RBD}$ patients with higher risk.

As expected, the performance of $\mathrm{iRBD}-\mathrm{OCI}$ on the NP test was also generally between those of iRBD-NC and iRBD-MCI groups, especially in the memory domain. For example, the mean scores of WLRT of the iRBD-OCI group were significantly lower than the NC group but significantly higher than the MCI group. One exception was CPT, wherein only the iRBD-OCI group showed a significant decrease compared to the iRBD-NC group, while the iRBD-MCI group demonstrated no changes. It is well known that MCI in alpha-synucleinopathy more likely displays impaired non-memory performance, particularly in the executive and attentive-visuospatial domains [32,33], unlike MCI in $\mathrm{AD}$ who have dominant memory problems [34]. However, since subjective cognitive complaints were more related to memory [35], participants having deficits only in non-memory tests (e.g. $\mathrm{CPT}$ ) might tend to be less self-aware of their cognitive decline and hence were evaluated as OCI instead of MCI. As expected, a higher ratio of non-amnestic subtypes were observed in the iRBD-OCI than in the iRBD-MCI group. The early detection of cognitive decline in the non-amnestic domain in iRBD should rely on not only the subjective complaint but also regular objective cognitive tests. It is in line with the current trend in psychiatric diagnosis that emphasizes the role objective tests [36]. For example, the latest revision of the Diagnostic and Statistical Manual of Mental Disorders 5th ed. included a substantial impairment in cognitive performance documented by standardized NP testing into the diagnostic criteria of major neurocognitive disorder. In addition, for patients with iRBD, who are at risk for neurodegenerative disorders, OCI more likely implies a non-negligible warning instead of an accidental drop in the score by chance.

The strengths of our study are that we recruited a relatively large sample of iRBD patients and performed complete NP tests in all the participants regardless of subjective cognitive complaints. In addition, we subdivided iRBD patients without MCI into with OCI group and with NC group. There are some limitations of our study. First, this study did not include healthy people as a control group. Therefore, the results should be interpreted carefully as between-group differences regarding the cognitive status among the iRBD patients. Even the least affected cognitive group, iRBD patients with NC, might have demonstrated EEG alteration underlying cerebral dysfunction related to iRBD. Second, although most of the participants underwent assessment including EEG and NP tests within one month after the diagnosis of $\mathrm{RBD}$, the duration of illness was not traceable and was not adjusted in the analyses. The extent of cerebral dysfunction and the concordant EEG alteration may be affected by the disease duration. Third, we did not consider the effects of medication on EEG, whereas clonazepam has been prescribed to most of the patients for their sleep behavior. Lastly, this study was a crosssectional study. A follow-up study with longitudinal assessment is needed to clarify whether patients with OCI are at more risk to develop MCI compared to patients with NC.

The current study demonstrated a gradual increase of slow wave brain activity in the order of iRBD-NC to iRBD-OCI to iRBD-MCI. We demonstrate the clinical importance of $\mathrm{OCI}$ in iRBD, which seems to be in the middle stage between $\mathrm{NC}$ and MCI, showing a moderate degree of EEG alteration between them. Clinicians should consider conducting NP tests for iRBD patients even before they report subjective cognitive problems, to detect early stages of cerebral dysfunction and cognitive impairment. We need further longitudinal study to examine whether iRBD patients with OCI are more likely to develop MCI or dementia than iRBD with NC in follow-up assessments.

\section{Acknowledgments}

None.

\section{Conflicts of Interest}

The authors have no financial conflicts of interest.

\section{Authors' Contribution}

Conceptualization: all authors. Data curation: Hyun JH, Hong JK. Formal analysis: Hyun JH. Supervision: Yoon IY. Writing_original draft: Hyun JH, Hong JK. Writing — review \& editing: all authors.

\section{REFERENCES}

1. Kaplan HI, Sadock BJ. Synopsis of psychiatry: behavioral sciences clinical psychiatry. 5th ed. Baltimore: Williams \& Wilkins Co 1988;556.

2. Jiang H, Huang J, Shen Y, Guo S, Wang L, Han C, et al. RBD and neurodegenerative diseases. Mol Neurobiol 2017;54:2997-3006.

3. Iranzo A, Molinuevo JL, Santamaría J, Serradell M, Martí MJ, Valldeoriola F, et al. Rapid-eye-movement sleep behaviour disorder as an early marker for a neurodegenerative disorder: a descriptive study. Lancet Neurol 2006;5:572-7.

4. Schenck CH, Boeve BF, Mahowald MW. Delayed emergence of a parkinsonian disorder or dementia in $81 \%$ of older men initially diagnosed with idiopathic rapid eye movement sleep behavior disorder: a 16-year update on a previously reported series. Sleep Med 2013;14:744-8.

5. Falup-Pecurariu C, Titova N, Ray Chaudhuri K. Editorial: Movement disorders and sleep - underlying mechanisms, clinical aspects and treatment. Front Neurol 2019;10:1034.

6. Gagnon JF, Vendette M, Postuma RB, Desjardins C, Massicotte-Mar- 
quez J, Panisset $\mathrm{M}$, et al. Mild cognitive impairment in rapid eye movement sleep behavior disorder and Parkinson's disease. Ann Neurol 2009; 66:39-47.

7. Fantini ML, Gagnon JF, Petit D, Rompré S, Décary A, Carrier J, et al. Slowing of electroencephalogram in rapid eye movement sleep behavior disorder. Ann Neurol 2003;53:774-80.

8. Iranzo A, Isetta V, Molinuevo JL, Serradell M, Navajas D, Farre R, et al. Electroencephalographic slowing heralds mild cognitive impairment in idiopathic REM sleep behavior disorder. Sleep Med 2010;11:534-9.

9. Rodrigues Brazète J, Montplaisir J, Petit D, Postuma RB, Bertrand JA, Génier Marchand D, et al. Electroencephalogram slowing in rapid eye movement sleep behavior disorder is associated with mild cognitive impairment. Sleep Med 2013;14:1059-63.

10. Fauzan N, Amran NH. Early detection of mild cognitive impairment, dementia and Alzheimer's using qEEG. European Journal of Interdisciplinary Studies 2015;1:149-53.

11. Kai T, Asai Y, Sakuma K, Koeda T, Nakashima K. Quantitative electroencephalogram analysis in dementia with Lewy bodies and Alzheimer's disease. J Neurol Sci 2005;237:89-95.

12. Bonanni L, Thomas A, Tiraboschi P, Perfetti B, Varanese S, Onofrj M. EEG comparisons in early Alzheimer's disease, dementia with Lewy bodies and Parkinson's disease with dementia patients with a 2-year follow-up. Brain 2008;131(Pt 3):690-705.

13. Rossini PM, Buscema M, Capriotti M, Grossi E, Rodriguez G, Del Percio $\mathrm{C}$, et al. Is it possible to automatically distinguish resting EEG data of normal elderly vs. mild cognitive impairment subjects with high degree of accuracy? Clin Neurophysiol 2008;119:1534-45.

14. Sasai T, Matsuura M, Inoue Y. Electroencephalographic findings related with mild cognitive impairment in idiopathic rapid eye movement sleep behavior disorder. Sleep 2013;36:1893-9.

15. Bang YR, Jeon HJ, Youn S, Yoon IY. Alterations of awake EEG in idiopathic REM sleep behavior disorder without cognitive impairment. Neurosci Lett 2017;637:64-9.

16. Edmonds EC, Delano-Wood L, Galasko DR, Salmon DP, Bondi MW. Subjective cognitive complaints contribute to misdiagnosis of mild cognitive impairment. J Int Neuropsychol Soc 2014;20:836-47.

17. Roberts JL, Clare L, Woods RT. Subjective memory complaints and awareness of memory functioning in mild cognitive impairment: a systematic review. Dement Geriatr Cogn Disord 2009;28:95-109.

18. Skov Neergaard J, Dragsbæk K, Christiansen C, Asser Karsdal M, Brix $\mathrm{S}$, Henriksen K. Objective cognitive impairment and progression to dementia in women: the prospective epidemiological risk factor study. J Prev Alzheimer Dis 2017;4:194-200.

19. Thorphy MJ. International classification of sleep disorder: diagnostic and coding manual. Rochester: Diagnostic Classification Steering Committee-American Sleep Disorder Association 1990.

20. Berry RB, Brooks R, Gamaldo CE, Harding SM, Lloyd RM, Marcus $\mathrm{CL}$, et al. The AASM manual for the scoring of sleep and associated events: rules, terminology and technical specifications. Ver. 2.0. Darien: American Academy of Sleep Medicine 2012;16-30.

21. Lee DY, Lee KU, Lee JH, Kim KW, Jhoo JH, Kim SY, et al. A normative study of the CERAD neuropsychological assessment battery in the Korean elderly. J Int Neuropsychol Soc 2004;10:72-81.

22. Lee JH, Lee KU, Lee DY, Kim KW, Jhoo JH, Kim JH, et al. Development of the Korean version of the Consortium to Establish a Registry for Alzheimer's Disease Assessment Packet (CERAD-K): clinical and neuropsychological assessment batteries. J Gerontol B Psychol Sci Soc Sci 2002;57:P47-53.

23. Wechsler DA. Wechsler memory scale-revised. New York: The Psychological Corporation 1987.

24. Stroop JR. Studies of interference in serial verbal reactions. J Exp Psychol 1935; 18:643.

25. Kim TH, Huh Y, Choe JY, Jeong JW, Park JH, Lee SB, et al. Korean version of frontal assessment battery: psychometric properties and normative data. Dement Geriatr Cogn Disord 2010;29:363-70.

26. Kim TH, Jhoo JH, Park JH, Kim JL, Ryu SH, Moon SW, et al. Korean version of mini mental status examination for dementia screening and its' short form. Psychiatry Investig 2010;7:102-8.

27. Winblad B, Palmer K, Kivipelto M, Jelic V, Fratiglioni L, Wahlund LO, et al. Mild cognitive impairment--beyond controversies, towards a consensus: report of the international working group on mild cognitive impairment. J Intern Med 2004;256:240-6.

28. Liang KY, Zeger SL. Longitudinal data analysis using generalized linear models. Biometrika 1986;73:13-22.

29. Choi JS, Park SM, Lee J, Hwang JY, Jung HY, Choi SW, et al. Restingstate beta and gamma activity in Internet addiction. Int $J$ Psychophysiol 2013;89:328-33.

30. Claassen J, Hirsch LJ, Kreiter KT, Du EY, Connolly ES, Emerson RG, et al. Quantitative continuous EEG for detecting delayed cerebral ischemia in patients with poor-grade subarachnoid hemorrhage. Clin Neurophysiol 2004;115:2699-710.

31. Son KL, Choi JS, Lee J, Park SM, Lim JA, Lee JY, et al. Neurophysiological features of Internet gaming disorder and alcohol use disorder: a resting-state EEG study. Transl Psychiatry 2015;5:e628.

32. Monastero R, Di Fiore P, Ventimiglia GD, Ventimiglia CC, Battaglini I, Camarda R, et al. Prevalence and profile of mild cognitive impairment in Parkinson's disease. Neurodegener Dis 2012;10:187-90.

33. Pal A, Biswas A, Pandit A, Roy A, Guin D, Gangopadhyay G, et al. Study of visuospatial skill in patients with dementia. Ann Indian Acad Neurol 2016;19:83-8.

34. Albert MS, DeKosky ST, Dickson D, Dubois B, Feldman HH, Fox NC, et al. The diagnosis of mild cognitive impairment due to Alzheimer's disease: recommendations from the National Institute on Aging-Alzheimer's Association workgroups on diagnostic guidelines for Alzheimer's disease. Alzheimers Dement 2011;7:270-9.

35. Burmester B, Leathem J, Merrick P. Subjective cognitive complaints and objective cognitive function in aging: a systematic review and metaanalysis of recent cross-sectional findings. Neuropsychol Rev 2016;26: 376-93.

36. American Psychiatric Association. Desk reference to the diagnostic criteria from DSM-5(TM). Washington: American Psychiatric Publishing, Inc. 2014;299-300. 